\title{
TOLERÂNCIA DE PLANTAS JOVENS DE CAFÉ A HERBICIDAS APLICADOS ISOLADAMENTE OU EM MISTURA COM
} O FERTILIZANTE FERTIACTYL

\section{TOLERANCE OF YOUNG COFFEE PLANTS TO HERBICIDE APPLIED ALONE OR IN COMBINATION WITH FERTIACTYL}

Jefferson Luiz Marciano do Nascimento ${ }^{\mathrm{a}}$, Gustavo Antônio Mendes Pereira ${ }^{\mathrm{a} *}$, Rodrigo Cabral Adriano ${ }^{\mathrm{a}}$, Laís Franchini Pucci ${ }^{\mathrm{a}}$, Lucas Heringer Barcellos Júnior $^{\mathrm{a}}$, Lino Roberto Ferreira ${ }^{\mathrm{a}}$

${ }^{\mathrm{a}}$ Departamento de Fitotecnia, Universidade Federal de Viçosa, Minas Gerais, Brasil. bepartamento de Agronomia, Universidade Federal de Viçosa, Minas Gerais, Brasil.

*Autor correspondente: gustavogamp@hotmail.com.

\section{INFORMAÇÕES DO ARTIGO}

\section{Histórico do artigo:}

Recebido: 07 Agosto 2019.

Aceito: 08 Abril 2020.

Publicado: 13 Abril 2020.

\section{Palavras-chave/Keywords:}

Controle químico/ Chemical control. Herbicidas pré-emergentes/ Preemergence herbicides.

Protetor químico/ Chemical protector. Seletividade/ Selectivity.

\section{Financiamento:}

CAPES - Coordenação de Aperfeiçoamento de Pessoal de Nível Superior.

Direito Autoral: Este é um artigo de acesso aberto distribuído sob os termos da Licença Creative Commons, que permite uso, distribuição e reprodução irrestritos em qualquer meio, desde que o autor e a fonte originais sejam creditados.

\section{Citação deste artigo:}

NASCIMENTO, J. L. M.; PEREIRA, G. A. M.; ADRIANO, R. C.; PUCCI, L. F.; BARCELLOS JÚNIOR, L. H.; FERREIRA， L. R. Tolerância de plantas jovens de café a herbicidas aplicados isoladamente ou em mistura com o fertilizante fertiactyl. Revista Brasileira de Herbicidas, v. 18, n. 4. 2019.

\section{RESUMO}

O controle de plantas daninhas na cultura do cafeeiro é dificultado pela escassez de herbicidas seletivos para a cultura em fase de implantação. Uma alternativa para contornar os danos causados pelos herbicidas é o uso de protetores químicos com capacidade de minimizar a intoxicação dos herbicidas. Objetivou-se avaliar a seletividade de herbicidas aplicados isoladamente ou em mistura no tanque com o Fertiactyl Sweet ${ }^{\circledR}$ para plantas de café recém-transplantadas. O experimento foi realizado em vasos em delineamento inteiramente casualizado, em esquema fatorial $10 \times 2$, com quatro repetições. $O$ primeiro fator correspondeu a nove herbicidas: (oxyfluorfen; sulfentrazone; flumioxazin; [isoxaflutole + indaziflam]; isoxaflutole; indaziflam; [metribuzin + indaziflam]; chlorimuron-etílico; e metsulfuron-metílico) e o controle sem aplicação de herbicida; o segundo fator constou da presença ou ausência do Fertiactyl Sweet. Os tratamentos foram aplicados em "over the top" aos 30 dias após o transplantio. De 7 aos 172 dias após a aplicação (DAA), avaliou-se a porcentagem de intoxicação. E aos 172 DAA, mensurou-se as variáveis morfológicas das plantas. A adição do Fertiactyl Sweet junto à calda dos herbicidas não modificou a tolerância das plantas de café aos herbicidas. Os herbicidas mais tolerantes para o café foram o oxyfluorfen e o sulfentrazone. A mistura comercial indaziflam + metribuzin causou a morte das mudas de café. O Fertiactyl Sweet ${ }^{\circledR}$ não reduziu a intoxicação das mudas de café arábica (Catuaí vermelho - linhagem 44) causada pelos herbicidas estudados. Os herbicidas metsulfuron-metílico e oxyfluorfen foram os mais tolerados pelas plantas de café recém-transplantadas.

\section{ABSTRACT}

The control of weeds in the coffee crop is hampered by the scarcity of selective herbicides for the growing crop. An alternative to circumvent the damage caused by herbicides is the use of chemical protectors with the ability to minimize herbicide intoxication. The objective was to evaluate the selectivity of herbicides applied alone or in a mixture in the tank with Fertiactyl Sweet ${ }^{\circledR}$ for recently transplanted coffee plants. The experiment was carried out in pots in a completely randomized design, in a 10x2 factorial scheme, with four replications. The first factor corresponded to nine herbicides: (oxyfluorfen; sulfentrazone; flumioxazin; [isoxaflutole + indaziflam]; isoxaflutole; indaziflam; [metribuzin + indaziflam]; chlorimuron-ethyl; and metsulfuron-methyl) and control without herbicide application; the second factor was the presence or absence of Fertiactyl Sweet. The treatments were applied "over the top" at 30 days after transplanting. From 7 to 172 days after application (DAA), the percentage of intoxication was evaluated. And at $172 \mathrm{DAA}$, the morphological variables of the plants were measured. The addition of Fertiactyl Sweet next to the herbicide syrup did not change the tolerance of coffee plants to herbicides. The most tolerant herbicides for coffee were oxyfluorfen and sulfentrazone. The commercial mixture indaziflam + metribuzin caused the death of the coffee seedlings. Fertiactyl Sweet ${ }^{\circledR} \operatorname{did}$ not reduce the poisoning of Arabica coffee seedlings (Catuaí Vermelho - lineage 44) caused by the studied herbicides. The herbicides metsulfuron-methyl and oxyfluorfen were the most tolerated by recently transplanted coffee plants. 


\section{Introdução}

O café (Coffea sp.) é uma commodity muito importante para economia nacional brasileira (CONAB, 2020). O país é o maior produtor, exportador e consumidor desta cultura, com cerca de $32 \%$ da produção mundial. Estima-se que o país possui 2.209 hectares de área plantada entre as espécies Arábica e Conilon, e produção de 49,67 milhões de sacas, sendo Minas Gerais o estado mais atuante, com 1.180 hectares plantados e produção de cerca de 28,5 milhões de sacas ao ano (CONAB, 2020).

Em culturas perenes como o café, os tratos culturais são mais complexos, pois são necessárias diferenciações entre as fases de crescimento inicial e produção das lavouras. O manejo das plantas daninhas nesta cultura é dividido em duas principias etapas, até os dois anos, chamada de lavoura jovem e após os dois anos, conhecida como manejo em café adulto. O manejo na lavoura jovem é o mais importante e complexo, pois, a cultura apresenta crescimento inicial muito lento e espaçamento largo, o que impossibilita a lavoura de formar folhagem capaz de suprimir o crescimento das plantas daninhas (FIALHO et al., 2010).

O manejo das plantas daninhas em ambos os lados na linha de plantio das mudas de café até o segundo ano pósplantio é essencial para o desenvolvimento da cultura. Araújo et al. (2012), concluíram que a faixa ideal que deve ser mantida livre de competição aumenta à medida que as plantas de café envelhecem. A manutenção desta faixa livre pode ser realizada com o uso de herbicidas seletivos aplicados em pré ou pós-emergência das espécies daninhas, ou através da utilização de herbicidas não-seletivos, que por sua vez devem ser aplicados em jato dirigido, evitando-se o contato das gotas pulverizadas com as plantas de café (RONCHI; SILVA; FERREIRA, 2001).

Atualmente, existem 30 ingredientes ativos com ação herbicida registrados para uso na cafeicultura (AGROFIT, 2020). Porém, poucos deles são comprovadamente seletivos ao cafeeiro. Dentre os produtos ou misturas de herbicidas comercializados para uso em cafezais jovens, seja em pré ou pós-emergência das plantas daninhas, têm-se o carfentrazone e o oxyfluorfen, sendo este último utilizado em pré-emergência (RODRIGUES; ALMEIDA, 2018).

Considerando a pouca disponibilidade de herbicidas disponíveis para o controle de plantas daninhas em espécies jovens de café é imprescindível a busca por alternativas que maximizem a eficácia desse método de controle. A intoxicação das culturas por herbicidas é um problema frequente, seja pela inadequação das doses, tolerância da cultivar ao herbicida e inadequada tecnologia de aplicação (MAGALHÃES et al., 2012). Protetores químicos podem ser utilizados para potencializar a tolerância de culturas reduzindo os efeitos tóxicos dos herbicidas. Também conhecidos como safeners ou antídotos, estas substâncias estão relacionadas à redução da absorção e/ou translocação, a metabolização e a competição com o local de ação dos herbicidas. Uma de suas atribuições é permitir a utilização de herbicidas não seletivos às culturas (GALON et al., 2011). O caráter protetor do Fertiactyl apesar de ser um fertilizante foliar, vem sendo pesquisado para minimizar os efeitos tóxicos causados por herbicidas, com resultados positivos em algumas culturas como cenoura (SILVA, 2015), soja (SANTOS et al., 2015) e eucalipto (MACHADO et al., 2017a, MACHADO et al., 2017b; MACHADO et al., 2017c).

A utilização de protetores químicos é uma alternativa promissora para redução da fitotoxidade de herbicidas em plântulas de café, podendo reduzir a dificuldade de controle de plantas daninhas na fase inicial de implantação do cafezal devido a carência de herbicidas seletivos. Assim, objetivouse com este estudo avaliar a seletividade de herbicidas aplicados isoladamente ou em mistura com o Fertiactyl Sweet ${ }^{\circledR}$ para plantas de café recém-transplantadas.

\section{Material e Métodos}

O experimento foi conduzido em ambiente não protegido, entre os meses de outubro de 2016 a maio de 2017, em área pertencente a Universidade Federal de Viçosa em Viçosa-MG (Coordenadas UTM (m): 7.703.630 N, $720.570 \mathrm{E}$ ). As mudas foram transplantadas em vasos de 14 $\mathrm{dm}^{3}$ preenchidos com latossolo vermelho-amarelo da região de Viçosa, corrigido e adubado conforme a análise de solo (Quadro 1) e recomendação para a cultura. Cada unidade experimental foi composta por uma planta de café arábica (Catuaí vermelho - linhagem 44) com aproximadamente sete meses de idade.

Quadro 1. Características químicas e físicas do solo utilizado no experimento em Viçosa-MG.

\begin{tabular}{|c|c|c|c|c|c|c|c|c|c|}
\hline \multicolumn{10}{|c|}{ Análise Química } \\
\hline pH & $\mathbf{P}$ & $\mathbf{K}$ & $\mathrm{Ca}^{2+}$ & $\mathrm{Mg}^{2+}$ & $\mathbf{A l}^{3+}$ & $\mathbf{H}+\mathbf{A l}$ & SB & CTC (t) & CTC (T) \\
\hline $\mathrm{H}_{2} \mathrm{O}$ & \multicolumn{2}{|c|}{$\mathrm{mg} \mathrm{dm} \mathrm{m}^{-3}$} & \multicolumn{7}{|c|}{$\mathrm{cmol}_{\mathrm{c}} \mathrm{dm}^{-3}$} \\
\hline 6,53 & 5 & 73 & 4 & 0,5 & 0 & 1,2 & 4,69 & 4,69 & 5,89 \\
\hline $\mathbf{v}$ & $\mathbf{m}$ & MO & & & $\mathbf{Z n}$ & $\mathbf{F e}$ & Mn & $\mathrm{Cu}$ & B \\
\hline \multicolumn{2}{|c|}{$\%$} & dag $\mathrm{Kg}^{-1}$ & \multicolumn{3}{|c|}{$m g L^{-1}$} & \multicolumn{4}{|c|}{$\mathrm{mg} \mathrm{dm^{-3 }}$} \\
\hline 79,6 & 0 & 2,02 & & & 29,28 & 67,9 & 41,5 & 3,23 & - \\
\hline \multicolumn{10}{|c|}{ Análise física } \\
\hline \multicolumn{10}{|c|}{ Análise granulométrica e classificação textural } \\
\hline Argila & Silte & Areia & \multicolumn{7}{|c|}{ Classificação textural } \\
\hline \multicolumn{3}{|c|}{$\%$} & \multirow{2}{*}{\multicolumn{7}{|c|}{ Muito argilosa }} \\
\hline 61,2 & 6,4 & 32,4 & & & & & & & \\
\hline
\end{tabular}

Análise realizada no Laboratório de análises de solos da Universidade Federal de Viçosa. 
Utilizou-se o delineamento inteiramente casualizado (DIC), em esquema fatorial $10 \times 2$ com quatro repetições, sendo que o primeiro fator constou de nove herbicidas e um controle sem aplicação de herbicidas, e o segundo fator constou da presença ou ausência do Fertiactyl Sweet ${ }^{\circledR}$. Os herbicidas avaliados foram oxyfluorfen (Goal BR $\AA$, 230,4 g i.a. ha $^{-1}$, EC, Dow AgroSciences); sulfentrazone (Boral ${ }^{\circledR}$ $500 \mathrm{SC}, 250 \mathrm{~g}$ i.a. ha ${ }^{-1}$, SC, FMC); flumioxazin (Flumyzin ${ }^{\circledR}$ $500 \mathrm{SC}, 50$ g i.a. ha ${ }^{-1}, \mathrm{SC}$, IHARABRAS); [isoxaflutole + indaziflam] (Provencel ${ }^{\circledR} 750 \mathrm{WG}, 16,87 \mathrm{~g}^{-}$i.a. $\mathrm{ha}^{-1}$, WG, Bayer + Alion ${ }^{\circledR}, 33,75$ g i.a. ha ${ }^{-1}$, SC, Bayer); isoxaflutole ((Provencel ${ }^{\circledR} 750$ WG, 112,5 g i.a. ha ${ }^{-1}$, WG, Bayer); indaziflam (Alion ${ }^{\circledR}, 37,5 \mathrm{~g}$ i.a. ha ${ }^{-1}, \mathrm{SC}$, Bayer); [metribuzin + indaziflam] (Alion Pro $\AA, 921,6+2,81$ g i. a. ha ${ }^{-1}, \mathrm{SC}$, Bayer); chlorimuron-etílico (Classic $₫, 6,25$ g i.a. ha ${ }^{-1}$, WG, DuPont) e metsulfuron-metílico (Ally® , 3,6 g i.a. ha ${ }^{-1}$,WG, DuPont). A dose do Fertiactyl Sweet ${ }^{\circledR}$ foi de $3 \mathrm{~L} \mathrm{ha}^{-1}$ do produto comercial, escolhida de acordo com ensaios preliminares.

Aos 30 dias após o transplantio, quando as plantas estavam com aproximadamente $11 \mathrm{~cm}$ de altura (de dois a três pares de folhas), foi realizada a aplicação dos herbicidas, cobrindo toda a planta, ou seja, a aplicação foi "over the top". Utilizou-se um pulverizador costal munido de barra com dois bicos com pontas tipo leque TTI 11002 , espaçados de $0,5 \mathrm{~m}$ entre si, operando a $250 \mathrm{kPa}$ de pressão e com volume de calda correspondente a $150 \mathrm{~L} \mathrm{ha}^{-1}$. No momento da aplicação a temperatura ambiente era $27,1^{\circ} \mathrm{C}$, a umidade relativa do ar $66 \%$ e a velocidade do vento 3,0 $\mathrm{Km} \mathrm{h}^{-1}$. Após a aplicação em "over the top", as plantas permaneceram protegidas do contato com água de chuva ou irrigação durante 24 horas, visando-se evitar a lavagem da calda aplicada.

A porcentagem de intoxicação das plantas foi avaliada aos 7, 25, 62, 75, 172 dias após a aplicação (DAA). A intoxicação foi quantificada por meio de avaliação visual, atribuindo-se notas percentuais em relação ao controle sem aplicação de herbicida (Tabela 1), sendo considerada zero a ausência de sintomas e 100 a morte da planta (SBCPD, 1995). Em ocasião da última avaliação (172 DAA), foram avaliadas a altura da planta (utilizando uma fita métrica), diâmetro do caule (utilizando um paquímetro digital), área foliar (por meio do medidor de área Licor Equipamentos modelo LI-3100), volume de raízes, número de nós, comprimento dos dois primeiros ramos plagiotrópicos (utilizando uma fita métrica) e o número de ramos plagiotrópicos. Posteriormente, folhas, caules e raízes foram acondicionados separadamente em sacos de papel e levados à estufa com circulação forçada de $\operatorname{ar}\left(65 \pm 3^{\circ} \mathrm{C}\right)$, até atingir massa constante para quantificação da massa seca.

Os dados obtidos foram submetidos à análise de variância pelo teste $F$, em caso de significância, as médias foram comparadas pelo teste de Tukey ao nível de $5 \%$ de significância.

Tabela 1. Escala de notas para avaliação visual de intoxicação sobre as plantas de café.

\begin{tabular}{|c|c|c|}
\hline CONCEITO & NOTAS & OBSERVAÇÃO \\
\hline Muito leve & $0-5$ & $\begin{array}{c}\text { Sintomas fracos ou pouco evidentes. Nota zero quando não se observam } \\
\text { quaisquer alterações na planta. }\end{array}$ \\
\hline Leve & $6-10$ & Sintomas nítidos, de baixa intensidade. \\
\hline Moderada & $11-20$ & Sintomas nítidos, mais intensos que na classe anterior. \\
\hline Aceitável & $21-35$ & Sintomas pronunciados, mas totalmente tolerados pela planta. \\
\hline Preocupante & $36-45$ & $\begin{array}{c}\text { Sintomas mais drásticos que na categoria anterior, mas ainda passíveis de } \\
\text { recuperação. }\end{array}$ \\
\hline Alta & $46-60$ & Danos irreversíveis, com redução drástica no desenvolvimento da planta. \\
\hline Muito alta & $61-100$ & Danos irreversíveis muito severos. Nota cem para morte da planta. \\
\hline
\end{tabular}

Adaptado de SBCPD (1995).

\section{Resultados e Discussão}

Verificou-se que não houve efeito significativo do Fertiactyl Sweet ${ }^{\circledR}$, assim como também da interação Fertiactyl $\mathrm{x}$ herbicidas para as características avaliadas nas plantas de café (Tabelas 2, 3 e 4). Este resultado mostra que o Fertiactyl Sweet ${ }^{\circledR}$ não interagiu com os herbicidas testados e não modificou a tolerância das plantas a estes herbicidas (Tabela 2). Todavia, houve efeito dos herbicidas para as variáveis, intoxicação das plantas, volume de raízes, massa seca de raízes, folhas, caule, área foliar, variáveis altura, diâmetro do caule, número de nós, número de ramos plagiotrópicos e comprimento médio dos dois primeiros ramos plagiotrópicos (Tabelas 2, 3 e 4). A mistura entre [metribuzin + indaziflam] foi incluída apenas na análise de variância da porcentagem de intoxicação, uma vez que eliminou todas as plantas do respectivo tratamento. 
NASCIMENTO, J. L. M et al.

Tabela 2. Resumo da análise de variância para a porcentagem de intoxicação das plantas de café aos 7, 25, 62, 75 e 172 DAA.

\begin{tabular}{lcccccc}
\hline \multirow{2}{*}{ FV } & GL & \multicolumn{5}{c}{ Quadrado Médio } \\
\cline { 3 - 7 } & & 7 DAA & 25 DAA & 62 DAA & 75 DAA & 172 DAA \\
\hline Herbicida & 9 & $1608,23^{*}$ & $4265,68^{*}$ & $4254,91^{*}$ & $5602,47^{*}$ & 0 \\
Fertiactyl & 1 & 2,38 & 10,62 & 12,92 & 24,26 & 0 \\
Herb*Fertiactyl & 9 & 20,49 & 7,45 & 5,21 & 6,43 & 0 \\
Resíduo & 60 & 73,59 & 46,22 & 41,77 & 30,47 & 0 \\
\hline \multicolumn{1}{c}{ C.V. $(\%)$} & & 32,09 & 15,50 & 18,93 & 23,09 & 0,00 \\
\hline
\end{tabular}

*Significativo a $(\mathrm{p} \leq 0,05)$ pelo teste $\mathrm{F}$.

Tabela 3. Resumo da análise de variância para as variáveis volume de raízes (VR), massa seca de raízes (MSR), folhas (MSF), caule (MSC) e área foliar (AF) das plantas de café aos 172 DAA.

\begin{tabular}{lcccccc}
\hline \multirow{2}{*}{ FV } & GL & \multicolumn{4}{c}{ Quadrado Médio } \\
\cline { 3 - 6 } & & VR & MSR & MSF & MSC & AF \\
\hline Herbicida & 8 & $1762,89^{*}$ & $41,36^{*}$ & $187^{*}$ & $68,3^{*}$ & $1777541,92^{*}$ \\
Fertiactyl & 1 & 703,12 & 10,58 & 6,48 & 0,27 & 61206,84 \\
Herb*Fertiactyl & 8 & 192,28 & 4,98 & 20,52 & 8,95 & 260529,70 \\
Resíduo & 54 & 745,99 & 10,34 & 48,31 & 14,78 & 559004,89 \\
\hline \multicolumn{1}{c}{ C.V. (\%) } & & 24,10 & 22,72 & 21,74 & 25,35 & 20,31 \\
\hline
\end{tabular}

* Significativo a $(\mathrm{p}<0,05)$ pelo teste $\mathrm{F}$.

Tabela 4. Resumo da análise de variância para as variáveis altura (ALT), diâmetro do caule (DC), número de nós (NN), número de ramos plagiotrópicos (NRP) e comprimento médio dos dois primeiros ramos plagiotrópicos (CRP) aos 172 DAA.

\begin{tabular}{lcccccc}
\hline & \multirow{2}{*}{ FV } & GL & \multicolumn{3}{c}{ Quadrado Médio } \\
\cline { 3 - 7 } & & ALT & DC & NN & NRP & CRP \\
\hline Herbicida & 8 & $64,69 *$ & $4,65^{*}$ & $159,26^{*}$ & $6,01^{*}$ & $28,1^{*}$ \\
Fertiactyl & 1 & 12,5 & 0,48 & 7,34 & 0,34 & 4,4 \\
Herb*Fertiactyl & 8 & 14,86 & 1,33 & 18,47 & 1,40 & 5,44 \\
Resíduo & 54 & 13,0 & 1,15 & 43,51 & 1,44 & 9,49 \\
\hline \multicolumn{1}{c}{ C.V. $(\%)$} & & 9,40 & 10,18 & 15,79 & 12,36 & 11,83 \\
\hline
\end{tabular}

* Significativo a $(\mathrm{p}<0,05)$ pelo teste $\mathrm{F}$.

As mudas de café arábica tratadas com os nove herbicidas mostraram diferentes níveis de intoxicação (Tabela 5). Aos 7 DAA, todos os herbicidas provocaram sintomas de intoxicação variando de leve a alto. Nesta avaliação, o isoxaflutole causou intoxicação moderada $(8,8 \%)$, enquanto que, o flumioxazin proporcionou alta intoxicação às plantas de café $(53,8 \%)$. Os demais herbicidas causaram graus de intoxicação intermediários (11 a 47\%). Observou-se que aos 25 DAA, a mistura comercial [metribuzin + indaziflam] eliminou todas as plantas, e que para os demais tratamentos, os sintomas de intoxicação se intensificaram, variando de 20,0 a $58,1 \%$. A partir dos 25 DAA, as plantas de café apresentaram sinais gradativos de recuperação dos efeitos tóxicos ocasionados pelos herbicidas, pois na última avaliação (172 DAA) já não havia sinais aparentes de intoxicação em nenhuma planta (Tabela 5). 
NASCIMENTO, J. L. M et al.

Tabela 5. Porcentagem de intoxicação (\%) de mudas de café arábica tratadas com herbicidas.

\begin{tabular}{|c|c|c|c|c|}
\hline \multirow{2}{*}{ Tratamento } & \multicolumn{4}{|c|}{ Dias após aplicação (DAA) $^{1 /}$} \\
\hline & 7 & 25 & 62 & 75 \\
\hline Controle & $0 \mathrm{a}$ & $0 \mathrm{a}$ & $0 \mathrm{a}$ & $0 \mathrm{a}$ \\
\hline Oxyfluorfen $^{2 /}$ & $41,2 \mathrm{~d}$ & $57,5 \mathrm{c}$ & $23,1 \mathrm{bcd}$ & $1,25 \mathrm{ab}$ \\
\hline Sulfentrazone ${ }^{3 /}$ & $47,5 \mathrm{~d}$ & $55,0 \mathrm{c}$ & 29,3 cde & $13,1 \mathrm{c}$ \\
\hline Flumioxazin ${ }^{4 /}$ & $53,8 \mathrm{~d}$ & $56 \mathrm{c}$ & $34,4 \mathrm{def}$ & $13,1 \mathrm{c}$ \\
\hline [Isoxaflutole+Indaziflam] $^{5 /}$ & $21,2 \mathrm{bc}$ & $58,1 \mathrm{c}$ & $45,0 \mathrm{e}$ & $28,1 \mathrm{~d}$ \\
\hline Isoxaflutole $^{6 /}$ & $8,8 \mathrm{ab}$ & $50,6 \mathrm{c}$ & $26,8 \mathrm{bcde}$ & $10,6 \mathrm{bc}$ \\
\hline Indaziflam $^{7 /}$ & $16,2 \mathrm{bc}$ & $54,3 \mathrm{c}$ & $40,6 \mathrm{ef}$ & $36,2 d$ \\
\hline Chlorimuron-etílico $^{8 /}$ & $16,9 \mathrm{bc}$ & $23,8 b$ & $15,6 \mathrm{bc}$ & $11,2 \mathrm{bc}$ \\
\hline Metsulfuron-metílico ${ }^{9 /}$ & $15 \mathrm{bc}$ & $20,0 b$ & $13,1 \mathrm{ab}$ & $0 \mathrm{a}$ \\
\hline [Metribuzin+Indaziflam] $^{10 /}$ & $26,2 \mathrm{c}$ & $100 \mathrm{~d}$ & $100 \mathrm{~g}$ & $100 \mathrm{e}$ \\
\hline C.V. $(\%)$ & 36,02 & 21,34 & 26,09 & 29,19 \\
\hline
\end{tabular}

${ }^{1 /}$ Médias seguidas por mesma letra na coluna não diferem entre si pelo teste de Tukey a $5 \%$ de significância; ${ }^{2 /}$ Goal; ${ }^{3 /}$ Boral;

${ }^{4 /}$ Flumyzin; ${ }^{5 /}$ Provence total; ${ }^{6 /}$ Fordor; ${ }^{7 /}$ Alion; ${ }^{8 /}$ Classic; ${ }^{9 /}$ Ally e ${ }^{10 /}$ Alion Pró.

Aos 25 DAA, a intoxicação causada pelo oxyfluorfen foi de $57,5 \%$, entretanto, na avaliação feita ao final do experimento (172 DAA) não foi verificado sinais de intoxicação (Tabela 5). Ronchi e Silva (2004), constataram que a aplicação de oxyfluorfen (480 $\mathrm{g} \mathrm{ha}^{-1}$ - metade da dose utilizada neste experimento) diretamente sobre o dossel do cafeeiro (C. arabica cv. Catuaí Vermelho) com seis a oito pares de folhas, aos 21 dias após o transplantio para o campo, causou leve toxicidade ao cafeeiro $(26,3 \%)$, sem comprometer o crescimento das mudas, avaliado aos 130 dias após o transplantio. O oxyfluorfen é um herbicida que inibe a PROTOX (HESS; WELLER, 2000). Os principais sintomas de injúrias visuais causados pelo oxyfluorfen às mudas de café são queimaduras e necroses espalhadas pela lâmina foliar (RONCHI: SILVA, 2003; MAGALHÃES et al., 2012), semelhantes aos resultados observados nesse estudo. Estas injúrias típicas são conferidas pela peroxidação de lipídios devido a presença de oxigênio reativo produzido pelos inibidores da PROTOX (HESS; WELLER, 2000).

O sulfentrazone causou $55 \%$ de intoxicação nas plantas de café aos 25 DAA, não apresentando intoxicação aos 172 DAA (Tabela 5). Os sintomas visuais de toxicidade conferidos pelo sulfentrazone foram queimaduras nas folhas, manchas cloróticas e encarquilhamento das folhas apicais, sintomas característicos dos herbicidas inibidores da PROTOX (HESS; WELLER, 2000). Magalhães et al. (2012), constataram níveis de intoxicação elevados em plantas de café de 52,4 e $61,8 \%$ quando utilizaram doses de 400 e $600 \mathrm{~g} \mathrm{ha}^{-1}$, respectivamente, aos 36 DAA. Avaliando a tolerância de mudas de café a herbicidas, Ronchi e Silva (2003), observaram que o sulfentrazone causou toxicidade moderada aos 16 DAA, denotada pelo enrugamento e necrose nas folhas mais novas, e que aquelas que surgiram posteriormente também apresentaram sintomas idênticos, porém com menor intensidade.
A recuperação das plantas de café tratadas com herbicidas inibidores da PROTOX pode estar relacionada com o acelerado metabolismo do herbicida nas plantas (comumente via citocromo P-450 ou conjugação com glutationa), podendo ocorrer também menor absorção foliar ou radicular, menor translocação, super produção ou insensibilidade enzimática e sequestro do herbicida. (CARVALHO; LÓPEZ-OVEJERO, 2008; GONÇALVES; CARVALHO, 2017).

O oxyfluorfen é registrado e recomendado para controle de plantas daninhas em aplicações em préemergência ou pós-emergência inicial na cultura do café após o transplantio, em jato dirigido sob o dossel das plantas. Já o sulfentrazone é recomendado apenas para cafeeiros adultos, em aplicação sob a copa das plantas (RODRIGUES; ALMEIDA, 2005). Contudo, os resultados desta pesquisa evidenciaram a possibilidade de uso do sulfentrazone em área total em lavouras de café em fase de implantação. Gonçalves e De Carvalho (2017) verificaram que o sulfentrazone possui características seletivas às plantas de café recém-transplantadas na dose de 400 g i.a. ha ${ }^{-1}$.

A mistura comercial [metribuzin+indaziflam] ocasionou a morte das plantas de café aos 25 DAA (Tabela 5). É possível que esse resultado tenha ocorrido pela ação do primeiro herbicida $\mathrm{O}$ mecanismo de ação do metribuzin caracteriza-se pela inibição do fotossistema II, que por meio de reações químicas inicia-se o processo de peroxidação das membranas, que culmina em necroses foliares (SILVA; FERREIRA; FERREIRA, 2009). Oliveira, Freitas e Vieira (2002), observaram reduções severas em massa seca, número de folhas, diâmetro do caule e altura de plantas de café após o tratamento com metribuzin. Em estudo conduzido por Ronchi e Silva (2003), viu-se que as mudas apresentaram intoxicação muito alta $(86,25 \%)$ aos 16 DAA após receberem $240 \mathrm{~g} \mathrm{ha}^{-1}$ do referido herbicida. 
As plantas de café que receberam os tratamentos contendo o herbicida indaziflam puro e a sua mistura comercial com o isoxaflutole não apresentavam sinais visíveis de sintomas aos 172 DAA, apesar da elevada intoxicação causada aos 25 DAA, de 54,3 e 58,1\%, respectivamente (Tabela 5). $\mathrm{O}$ indaziflam pertence à classe química denominada alkylazine, que por sua vez atua na biossíntese da parede celular, sendo considerado o mais potente inibidor desta estrutura já descoberto (MYERS et al., 2009; KAAPRO; HALL, 2012). Silva (2016), estudando o efeito da deriva do indaziflam sobre mudas de café conilon vitória incaper 8134 , verificou que $40 \%$ de deriva da dose recomendada para a cultura $\left(150 \mathrm{~g}\right.$ i.a. $\left.\mathrm{ha}^{-1}\right)$, causou intoxicação leve (20\%) aos 28 dias após a simulação da deriva.
O metsulfuron-metílico apresentou intoxicação moderada aos 7 DAA (15\%) e não foi observada sintomas de alteração nas plantas aos 75 DAA (Tabela 5). Em experimento conduzido por Ronchi e Silva (2004), constatou-se que o herbicida flazasulfuron pertencente ao mesmo grupo químico do metsulfuron-metílico sulfoniluréias (OLIVEIRA JR; CONSTANTIN; INOUE, 2011), causou intoxicação leve (25\%) e não prejudicou as variáveis altura de planta e diâmetro do caule do cafeeiro aos 130 DAA. Por outro lado, o chlorimuron-etílico, apesar de também integrar o grupo químico das sulfoniluréias, não causou intoxicação aos 172 DAA (Tabela 5) e afetou negativamente a maioria das variáveis avaliadas neste experimento (Tabelas 6 e 7).

Tabela 6. Médias do volume de raízes (VR), massa seca de raízes (MSR), de folhas (MSF), de caule (MSC) e área foliar (AF) das plantas de café, aos 172 DAA.

\begin{tabular}{|c|c|c|c|c|c|}
\hline \multirow{2}{*}{ Tratamento } & \multirow[b]{2}{*}{ VR (mL) } & \multicolumn{4}{|c|}{ Variáveis* } \\
\hline & & $\operatorname{MSR}(\mathrm{g})$ & $\operatorname{MSF}(\mathrm{g})$ & $\operatorname{MSC}(\mathrm{g})$ & $\mathrm{AF}\left(\mathrm{cm}^{2}\right)$ \\
\hline Controle & $145 \mathrm{a}$ & $19,31 \mathrm{a}$ & $40,30 \mathrm{a}$ & $21,29 \mathrm{a}$ & $4555,9 \mathrm{a}$ \\
\hline Oxyfluorfen & $114 \mathrm{ab}$ & $13,75 \mathrm{ab}$ & $36,29 \mathrm{ab}$ & $16,72 \mathrm{ab}$ & $4089,2 \mathrm{ab}$ \\
\hline Sulfentrazone & $118 \mathrm{ab}$ & $15,40 \mathrm{ab}$ & $35,51 \mathrm{abc}$ & $16,83 \mathrm{ab}$ & $3955,36 a b$ \\
\hline Flumioxazin & $102 b$ & $12,16 b$ & $30,12 \mathrm{abc}$ & $13,53 b$ & $3402,5 b$ \\
\hline Isoxaflutole+Indaziflam & $102 b$ & $12,33 b$ & $24,96 \mathrm{c}$ & $11,68 b$ & $2973,0 \mathrm{~b}$ \\
\hline Isoxaflutole & $95 b$ & $12,31 b$ & $29,76 \mathrm{abc}$ & $13,77 b$ & $3358,8 b$ \\
\hline Indaziflam & $112 \mathrm{ab}$ & $14,79 \mathrm{ab}$ & $27,50 \mathrm{bc}$ & $12,18 b$ & $3434,5 \mathrm{ab}$ \\
\hline Chlorimuron-etílico & $108 \mathrm{ab}$ & $12,81 \mathrm{~b}$ & $29,52 \mathrm{bc}$ & $15,37 \mathrm{ab}$ & $3566,1 \mathrm{ab}$ \\
\hline Metsulfuron-metílico & $124 \mathrm{ab}$ & $14,57 \mathrm{ab}$ & $33,72 \mathrm{abc}$ & $15,11 \mathrm{~b}$ & $3798,1 \mathrm{ab}$ \\
\hline C.V. $(\%)$ & 22,92 & 21,96 & 20,78 & 24,50 & 19,46 \\
\hline [metribuzin+indaziflam] & - & - & - & - & - \\
\hline
\end{tabular}

*Médias seguidas por mesma letra na coluna não diferem entre si pelo teste de Tukey a 5\% de significância.

Flumioxazin, isoxaflutole + indaziflam e isoxaflutole reduziram o volume de raízes em $29,7,29,7$ e $34,5 \%$ comparados ao controle sem aplicação de herbicidas, respectivamente (Tabela 7). Com menor desenvolvimento das raízes a absorção de água e nutrientes fica prejudicada, comprometendo o desenvolvimento inicial do cafeeiro (FRANÇA et al., 2010). Além disso, Flumioxazin, isoxaflutole + indaziflam, isoxaflutole e chlorimuron-etílico reduziram a massa seca de raízes quando comparado ao controle sem aplicação de herbicidas (Tabela 6). A massa seca de folhas foi influenciada de forma negativa pelos herbicidas [isoxaflutole + indaziflam], indaziflam e chlorimuron-etílico, havendo reduções de $38,1,31,8$ e $26,7 \%$, respectivamente em relação ao controle sem aplicação de herbicidas (Tabela 6). Estudos de seletividade verificaram que a aplicação de $15 \mathrm{~g}$ i.a. ha $^{-1}$ de chlorimuronetílico também diminuiu a massa seca das folhas de café
(GONÇALVES; DE CARVALHO, 2017). A MSC sofreu redução quando as plantas foram submetidas aos herbicidas, Flumioxazin, Isoxaflutole+Indaziflam, Isoxaflutole, Indaziflam e Metsulfuron-metílico.

Houve redução significativa da área foliar pela aplicação de flumioxazin, isoxaflutole + indaziflam e isoxaflutole $(25,3,34,7$ e $26,3 \%$, respectivamente) quando comparado ao controle sem aplicação de herbicidas (Tabela 6).Observa-se que não houve correlação entre a redução de área foliar com o aumento da intoxicação de plantas, o que indica que cada herbicida atua de forma diferente nas plantas, resultando em dados diferentes. Esta característica é um dos componentes de produção mais importantes para as plantas, uma vez que a produtividade está correlacionada com a fotossíntese, que depende da interceptação da energia luminosa, e esta depende dentre outros fatores, da área foliar (AQUINO et al., 2011). 
NASCIMENTO, J. L. M et al.

Tabela 7. Médias da altura (ALT), diâmetro do caule (DC), número de nós (NN), número de ramos plagiotrópicos (NRP) e comprimento médio dos dois primeiros ramos plagiotrópicos (CRP) aos 172 DAA.

\begin{tabular}{|c|c|c|c|c|c|}
\hline \multirow{2}{*}{ Tratamento } & \multicolumn{5}{|c|}{ Características* } \\
\hline & ALT (cm) & DC (mm) & $\mathbf{N N}$ & NRP & CRP (cm) \\
\hline Controle & $42,3 \mathrm{a}$ & $12,23 \mathrm{a}$ & $48,1 \mathrm{a}$ & $10,9 \mathrm{a}$ & $29,4 a$ \\
\hline Oxyfluorfen & $40,6 \mathrm{ab}$ & $10,68 \mathrm{ab}$ & $45,5 \mathrm{ab}$ & $11 \mathrm{a}$ & $27,7 \mathrm{ab}$ \\
\hline Sulfentrazone & $39,9 a b$ & $10,89 \mathrm{ab}$ & $44,8 \mathrm{abc}$ & $9,9 \mathrm{ab}$ & $27,4 \mathrm{ab}$ \\
\hline Flumioxazin & $39,2 \mathrm{ab}$ & $10,11 \mathrm{~b}$ & $36,4 \mathrm{bc}$ & $9,9 \mathrm{ab}$ & $25,7 \mathrm{ab}$ \\
\hline [Isoxaflutole+Indaziflam] & $35,9 \mathrm{ab}$ & $9,54 b$ & $35,1 \mathrm{c}$ & $8,4 \mathrm{~b}$ & $23,4 b$ \\
\hline Isoxaflutole & $37,6 a b c$ & $10,93 \mathrm{ab}$ & $40,9 \mathrm{abc}$ & $9,4 \mathrm{ab}$ & $25,2 \mathrm{ab}$ \\
\hline Indaziflam & $32,5 \mathrm{c}$ & $10,29 b$ & $38,5 \mathrm{abc}$ & $9,5 \mathrm{ab}$ & $24,5 b$ \\
\hline Chlorimuron-etílico & $39,0 \mathrm{ab}$ & $10,11 \mathrm{~b}$ & $41,5 \mathrm{abc}$ & $8,8 \mathrm{~b}$ & $24,8 \mathrm{ab}$ \\
\hline Metsulfuron-metílico & $38,3 \mathrm{abc}$ & $10,28 b$ & $45,1 \mathrm{abc}$ & $10 \mathrm{ab}$ & $26,1 \mathrm{ab}$ \\
\hline C.V. (\%) & 9,49 & 10,24 & 15,10 & 12,27 & 11,46 \\
\hline [Metribuzin+Indaziflam] & - & - & - & - & - \\
\hline
\end{tabular}

Médias seguidas por mesma letra na coluna não diferem entre si pelo teste de Tukey a 5\% de significância.

A altura de plantas foi significativamente prejudicada pelo tratamento indaziflam (redução de 23,2\%) em relação ao controle (Tabela 7). Os herbicidas flumioxazin, isoxaflutole + indaziflam, indaziflam, chlorimuron-etílico e metsulfuron-metílico causaram reduções significativas no diâmetro do caule, em relação ao controle sem aplicação de herbicidas (Tabela 7). Flumioxazin e isoxaflutole + indaziflam também reduziram o número de nós em 24,3 e $27 \%$, em relação ao controle, respectivamente (Tabela 7). Portanto, a aplicação destes dois herbicidas pode causar uma redução na produtividade, uma vez que o número de nós é um bom indicador da quantidade disponível de gemas produtivas, sendo um dos principais componentes de produtividade (BONOMO et al., 2004).

$\mathrm{O}$ número de ramos plagiotrópicos foi diminuído em 22,9 e 19,3\%, pelos herbicidas isoxaflutole + indaziflam e chlorimuron-etílico em relação ao controle, respectivamente, com relação inversamente proporcional ao ocorrido com a intoxicação de plantas (Tabela 7). Os herbicidas isoxaflutole + indaziflam e indaziflam causaram diminuições significativas no comprimento médio dos dois primeiros ramos plagiotrópicos, em 20,4 e 16,7\% quando comparados ao controle, respectivamente (Tabela 7), o que pode afetar negativamente a produtividade (CARVALHO et al., 2011).

\section{Conclusão}

Conclui-se que o Fertiactyl Sweet ${ }^{\circledR}$ não reduziu a intoxicação das mudas de café arábica (Catuaí vermelho linhagem 44) pelos herbicidas estudados. Os herbicidas metsulfuron-metílico e oxyfluorfen foram os mais tolerados pelas plantas de café recém-transplantadas. As plantas de café recém-transplantadas são sensíveis ao metribuzin+indaziflam, não sendo recomendado seu uso no ciclo inicial da cultura.

\section{Referências}

Agrofit. Ministério da Agricultura, Pecuária e Abastecimento. Agrofit, Consulta aberta. Disponível em: $<$ http://agrofit.agricultura.gov.br/agrofit_cons/ principal_agrofit_cons $>$. Acesso em: 10 abr. 2020.

Aquino, L. A.; Júnior V. C. S.; Guerra, J. V. S.; Costa, M. M. Estimativa da área foliar do girassol por método não destrutivo. Bragantia, v. 70, n. 4, p. 832-836, 2011.

Araújo, F.C.; Ronchi, C. P.; Almeida, W. L.; Silva, M. A. A.; Magalhães, C. E. O.; Good-god, P. I. V. Optimizing the width of strip weeding in Arabica coffee in relation to crop age. Planta Daninha, v. 30, n. 1, p. 129-138, 2012.

Bonomo, P.; Cruz, C. D.; Viana, J. M. S.; Pereira, A. A.; Oliveira, V. R. de; Carneiro, P. C. S. Avaliação de progênies obtidas de cruzamentos de descendentes do híbrido de Timor com as cultivares Catuaí Vermelho e Catuaí Amarelo. Bragantia, v. 63, p. 207-219, 2004.

Carvalho, S. J. P.; López-Ovejero, R. F. Resistência das plantas daninhas aos herbicidas inibidores da protox (Grupo E). In: Christoffoleti, P. J. (Coord.). Aspectos de resistência de plantas daninhas a herbicidas. Piracicaba: HRAC-BR, 2008, 3 ed., p. 69-77.

Carvalho, A. M.; Mendes, A. N.; Carvalho, G. R.; Botelho, C. E.; Gonçalves, F. A.; Ferreira, A. D. Correlação entre crescimento e produtividade de cultivares de café em diferentes regiões de Minas Gerais, Brasil. Pesquisa Agropecuária Brasileira, v. 45, n. 3, p. 269-275, 2011.

Fialho, C. M. T.; Silva, G. R.; Freitas, M. A. M.; França, A. C.; Mello, C. A. D.; SILVA, A. A. Competição de plantas 
daninhas com a cultura do café em duas épocas de infestação. Planta Daninha, v. 28, p. 969-978, 2010.

França, A. C.; Freitas, M. A. M.; Fialho, C. M. T.; Silva, A. A.; Reis, M. R.; Galon, L.; Victoria Filho, R. Crescimento de cultivares de café arábica submetidos a doses do glyphosate. Planta Daninha, v. 28, n. 3. 2010.

Galon, L.; Maciel, C. D. G.; Agostinetto, D.; Concenço, G.; Moraes, P. V. D. Seletividade de herbicidas às culturas pelo uso de protetores químicos. Revista Brasileira de Herbicidas, v. 10, n. 3, p. 291-304, 2011.

Gonçalves, M.F.; Carvalho, S.J.P. Seletividade do herbicida sulfentrazone em pós-emergência sobre mudas de café recém implantadas. Revista Agrogeoambiental, v. 9, n. 1, 2017.

Hess, F. D.; Weller, S. C. Inhibitors of protoporphyrinogen oxidase: diphenyl ethers and oxadiazon. In: Herbicide action. West Lafayette: Purdue University, p. 225-243, 2000 .

Kaapro, J.; Hall, J. Indaziflam, a new herbicide for preemergent control of weeds in turf, forestry, industrial vegetation and ornamentals. Pakistan Journal Weed Science Research, v. 18, n. esp., p. 267-270, 2012.

Machado, M. S.; Ferreira, L. R.; Paula, J. L.; Pereira, G. A. M.; Gonçalves, V. A. Use of liquid fertilizer to reduce the phytotoxic effects of glyphosate on eucalyptus. Revista Caatinga, v. 30, p. 730-737, 2017a.

Machado, M. S., Ferreira, L. R., Pereira, G. A. M., Gonçalves, V. A., Paixão, G. P. Protective Effect on Eucalyptus Plants and Signal Grass Control with a Tank Mixture of Glyphosate and Liquid fertilizer. Planta Daninha, v. 35, p. 1-8, 2017 b.

Machado, M. S., Ferreira, L. R., Pereira, G. A. M., Paula, J. L., Paixão, G. P., Freitas, P. M. Fertiactyl Pós ${ }^{\circledR}$ como protetor do eucalipto submetido à aplicação de glyphosate. Revista Brasileira de Ciências Agrarias, v. 12, p. 194-201, 2017c.

Magalhães, C .E. O.; Ronchi, C. P.; Ruas, R. A. A.; Silva. M. A. A.; Araújo, F. C.; Almeida, W. L. Seletividade e controle de plantas daninhas com oxyfluorfen e sulfentrazone na implantação de lavoura de café. Planta Daninha, v. 30, n. 3, p. 607-616, 2012.

Myers, D. F.; Hanrahan, R.; Michel, J.; Monke, B.; Mudge, L.; Olsen, C.; Spak, D. Indaziflam/BCS AA170717 - a new herbicide for preemergent control of grasses and broadleaves in turf and ornamentals. Proceeding South Society Weed Science Abstracts, v. 62, p. 393, 2009.

Oliveira JR, R. S.; Constantin, J.; Inoue, M. H. Mecanismo de ação de herbicidas. In: Oliveira JR, R. S.; Constantin, J.; Inoue, M. H. Biologia e manejo de plantas daninhas.
Ominipax, Curitiba-PR, Brasil, p. 141-192, 2011.

Oliveira, A. R.; Freitas, S. P.; Vieira, H. D. Tolerância de mudas de café aos herbicidas utilizados no controle de plantas daninhas. In: Congresso brasileiro da ciência das plantas daninhas, 23, 2002, Gramado, RS. Resumos... Londrina, PR: SBCPD/EMBRAPA Clima Temperado, p. 462, 2002.

Rodrigues, B. N.; Almeida, F. S. Guia de herbicidas. 7. ed. Londrina: PbR, 648 p. 2018.

Ronchi, C. P.; Silva, A. A. Tolerância de mudas de café a herbicidas aplicados em pós-emergência. Planta Daninha, v. 21, n. 3, p. 421-426, 2003.

Ronchi, C. P.; Silva, A. A. Weed control in young coffee plantations through post-emergence herbicide application onto total area. Planta Daninha, v. 22, n. 4, p. 607-615, 2004.

Ronchi, C. P.; Silva, A. A.; Ferreira, L. R. Manejo de plantas daninhas em lavouras de café. Viçosa, MG: Universidade Federal de Viçosa, Departamento de Fitopatologia, 94 p. 2001.

Santos, A. C. M.; Souza, M. A. S.; Freitas, G. A.; Santos, P. S.S.; Silva, R. R. Substância húmica na redução da fitotoxicidade dos herbicidas roundup ready + lactofene na cultura da soja. Tecnologia Ciência Agropecuária, v. 9, n. 3 , p. $35-41,2015$

Silva, A. A.; Ferreira, F. A.; Ferreira, L. R. Herbicidas: Classificação e Mecanismos de Ação. In: SILVA, A. A.; SILVA, J. F. Tópicos em manejo de plantas daninhas. Viçosa, MG: Universidade Federal de Viçosa, p. 83-148, 2009.

Silva, G. S. Fertilizantes foliares como atenuadores de intoxicação da cenoura pelo metribuzin. 2015. 62 p. Dissertação (Mestrado em Fitotecnia) - Universidade Federal de Viçosa, UFV, Viçosa, 2015.

Silva, W. C. Desempenho dos herbicidas indaziflam e glifosato na cultura do café Conilon. 2016. 60 p. Dissertação (Mestrado em Produção Vegetal) Universidade Estadual do Norte Fluminense Darcy Ribeiro, Campos dos Goytacazes, Rio de Janeiro, 2016.

SOCIEDADE BRASILEIRA DA CIÊNCIA DAS PLANTAS DANINHAS - SBCPD. Procedimentos para instalação, avaliação e análise de experimentos com herbicidas. Londrina, 42 p. 1995. 